\title{
Tracking a Surrogate Hazardous Agent (Rhodamine Dye) in a Coastal Ocean Environment Using In Situ Measurements and Concentration Estimates Derived from Drone Images
}

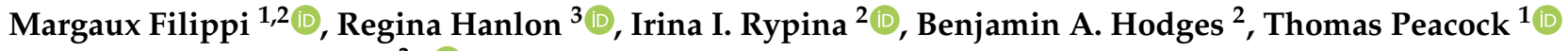 \\ and David G. Schmale III ${ }^{3, * \text { (D) }}$ \\ 1 Department of Mechanical Engineering, Massachusetts Institute of Technology, Cambridge, MA 02139, USA \\ margaux@mit.edu (M.F.); tomp@mit.edu (T.P.) \\ 2 Physical Oceanography Department, Woods Hole Oceanographic Institution, Woods Hole, MA 02543, USA; \\ irypina@whoi.edu (I.I.R.); bhodges@whoi.edu (B.A.H.) \\ 3 School of Plant and Environmental Sciences, Virginia Tech, Blacksburg, VA 24061, USA; rhanlon@vt.edu \\ * Correspondence: dschmale@vt.edu
}

\section{check for}

updates

Citation: Filippi, M.; Hanlon, R.; Rypina, I.I.; Hodges, B.A.; Peacock, T.; Schmale, D.G., III. Tracking a Surrogate Hazardous Agent (Rhodamine Dye) in a Coastal Ocean Environment Using In Situ Measurements and Concentration Estimates Derived from Drone Images. Remote Sens. 2021, 13, 4415. https://doi.org/10.3390/rs13214415

Academic Editor: Yukiharu Hisaki

Received: 14 September 2021

Accepted: 15 October 2021

Published: 2 November 2021

Publisher's Note: MDPI stays neutral with regard to jurisdictional claims in published maps and institutional affiliations.

Copyright: (c) 2020 by the authors. Licensee MDPI, Basel, Switzerland. This article is an open access article distributed under the terms and conditions of the Creative Commons Attribution (CC BY) license (https:/ / creativecommons.org/licenses/by/ $4.0 /)$.

\begin{abstract}
New tools and technology are needed to track hazardous agents such as oil and red tides in our oceans. Rhodamine dye (a surrogate hazardous agent) was released into the Atlantic ocean in August 2018, and experiments were conducted to track the movement of the dye near the water surface within three hours following the release. A DrOne Water Sampling SystEm (DOWSE), consisting of a 3D-printed sampling device tethered to a drone, was used to collect 26 water samples at different locations around the dye plume. Rhodamine concentrations were measured from the drone water samples using a fluorometer and ranged from 1 to $93 \mathrm{ppb}$. Dye images were taken during the drone-sampling of surface water containing dye and at about $10 \mathrm{~m}$ above the sampling point. These images were post-processed to estimate dye concentrations across the sampling domain. A comparison of calibrated heat maps showed that the altitude images yielded dye distributions that were qualitatively similar to those from images taken near the ocean surface. Moreover, the association between red ratios and dye concentrations yielded trendlines explaining up to $67 \%$ of the variation. Drones may be used to detect, track and assist in mitigating hazardous agents in the future.
\end{abstract}

Keywords: UAS; drone; fluorescent dye; rhodamine; transport; hazardous agents; plume; unmanned systems

\section{Introduction}

New tools and technology are needed to understand the transport of hazardous agents and track them in our oceans and waterways [1]. Such technology can be used to determine when people can safely return to their homes and work after a natural disaster and when it is safe for first responders to conduct rescue and recovery efforts in and around contaminated floodwaters. Modeling efforts based on the identification or prediction of hazards [2,3] can be used to prioritize the deployment of unmanned assets, positioning unmanned systems at the right time and place to do dull, dirty, and dangerous missions [4].

Powers et al. [5] showed the value of coordinated unmanned systems in the air and water to track the plume of a fluorescent dye. A small quadcopter was used to capture images of the plume, and heatmaps were generated from drone images to estimate plume concentrations. An unmanned surface vehicle (USV) equipped with an onboard fluorometer was used to provide real-time measurements of the dye in the plume while the drone was capturing the images. Other studies have found utility in the use of unmanned aerial systems (UASs) to estimate loads of hazardous agents such as harmful algal blooms (HABs) [6]. Consumer-grade drones, or UASs, have been increasingly used in oceanography over the past two decades, with technological advances allowing light-weight sensors 
and instruments to be mounted on small UAS [7]. The U.S. Integrated Ocean Observing System (IOOS) has been incorporating measurements from UAS-mounted sensors for over 15 years [8]. UASs have also been used in coral ecology [9], hydrography [10-13] and to track pathogens in the air [14]. Benson et al. [15] developed a DrOne Water Sampling SystEm (DOWSE) to study microorganisms in freshwater habitats. This system This system consisted of a 3D-printed sampling device tethered to a drone, and was used to collect water samples at the surface of eight different lakes in Austria.

Powers et al. [5] used an UAS to detect an artificial dye plume (fluorescein) in a freshwater lake. The UAS images were used a posteriori to construct the heat maps and were compared to concentration measurements collected from a fluorescence sensor mounted on the USV. The heat maps were generated from the images' red, blue and green (RGB) decomposition, using the fluorescein RGB value of $(100,200,60)$ as a reference. The images were scaled as a percentage of this maximum intensity and then scaled based on minimum and maximum dye concentrations from the USV fluorescein sensor. Extending this method to the open ocean remains challenging, however, in part due to the sun reflection induced by the surface wave state, a problem often encountered in the literature [16,17]. Zeng et al. [17] investigated how images acquired with UAS can be used to evaluate water quality and which environmental variables affected the results; they found that surface wave height and sun glint were particularly challenging and a significant factor in the accuracy of regression models. The wave height can also be an obstacle to UAS navigation. Flying at higher altitudes is much safer and more economical than having to lower the drone close to the ocean surface $(<10 \mathrm{~m})$. Moreover, open ocean plume studies could find unique applications from swarms of UASs tracking plumes at higher altitudes $(>100 \mathrm{~m})$. To our knowledge, the effect of altitude on the accuracy of dye concentration heat maps has not yet been assessed in detail. Another barrier to UAS-based plume studies is the cost associated with photogrammetric processing of the images; though commercial software are available, such as Agisoft PhotoScan used in $[9,16]$, their cost is a significant barrier to widespread adoption.

The present study took place as part of a larger project studying Lagrangian dynamics in the coastal ocean. Two plumes of fluorescent rhodamine WT dye were released south of Martha's Vineyard, Massachusetts, concurrently with surface and subsurface drifters. Rhodamine WT was chosen as a surrogate hazardous agent because of its low environmental impact. Field et al. [18] studied the ecotoxicity of fluorescent dyes, including Rhodamine WT, and found low levels of concern for concentrations under $2 \mathrm{mg} \cdot \mathrm{L}^{-1}$, a threshold orders of magnitude higher than the concentrations used in this study. Earlier work by Parker [19] tested the toxicity of Rhodamine WT dye on the larval development of oysters and on juvenile salmon and trout; with concentrations up to $10 \mathrm{mg} \cdot \mathrm{L}^{-1}$ for oysters and $375 \mathrm{mg} \cdot \mathrm{L}^{-1}$ for fish, no mortalities or abnormalities were observed. Dye plumes are frequently released to conduct dispersion studies in oceanography [20-22]. In the past few years, several studies investigated how to estimate concentrations of rhodamine dye using images from a small UAS. Legleiter et al. [23] stirred dye in experimental tanks to evaluate the potential to map dye patterns via remote sensing in turbid rivers. Baek et al. [24] released dye in an experimental channel and built artificial neural networks to construct the relationship between UAS images and dye concentrations. Both studies were conducted from land, and ground control points (GCPs) were used for altitude calibration and orientation of the UAS. To our knowledge, these investigations have not yet been extended to a coastal or open ocean environment, where the field conditions present significant challenges. The deep learning approach of Baek et al. [24], for example, required high volumes of images to build reliable neural networks. The authors were able to record between 13,440 and 23,688 frames per experiment, but these high numbers are much harder to obtain when launching UAS from a research vessel in the open ocean.

In this paper, we describe a unique study using drones to examine the transport of a kilometer-scale dye release in an open ocean environment, where the field conditions presented significant challenges. A drone water-sampling system was used to sample and 
image rhodamine dye plume released in the Atlantic Ocean in 2018. Images of the dye plume were taken, and rhodamine concentrations were determined onboard the research vessel using a fluorometer immediately after the samples were collected. The specific objectives of this study were to: (1) track the movement of a rhodamine dye plume within three hours following a rhodamine dye release in situ using measurements from unmanned systems, (2) estimate concentrations of rhodamine dye from post-processed drone images taken at the sea surface and at altitude, and without using expensive commercial software, and (3) compare concentrations of rhodamine dye among drone water samples and droneimage estimation methods. Since the images were taken from a UAS, standard homography methods could not be applied because the sea surface was photographed from different angles and altitudes.

\section{Materials and Methods}

\subsection{Rhodamine Dye Release}

A kilometer-scale Rhodamine dye release was planned and then carried out on 16 August 2018, south of Martha's Vineyard in Massachusetts, USA. The specific location was chosen because real-time forecasts predicted the occurrence of a salinity intrusion around this location. The output from the forecasts for the Martha's Vineyard shelf, along with ocean physics data, including temperature and salinity conditions, as well as the atmospheric and tidal forcing that were assimilated in the models, can be found at http:/ / mseas.mit.edu/Sea_exercises/NSF_ALPHA/2018/ (accessed on 15 October 2021). Drifter trajectories that were in the vicinity in of the study site during the release indicated an average mean surface velocity of $0.35 \mathrm{~m} \mathrm{~s}^{-1}$.

The location of the plume study is shown in Figure 1. Our research team worked on board R/V Connecticut (R/V UCONN), a 27.43-m steel research vessel, owned and operated by the Department of Marine Sciences, University of Connecticut.

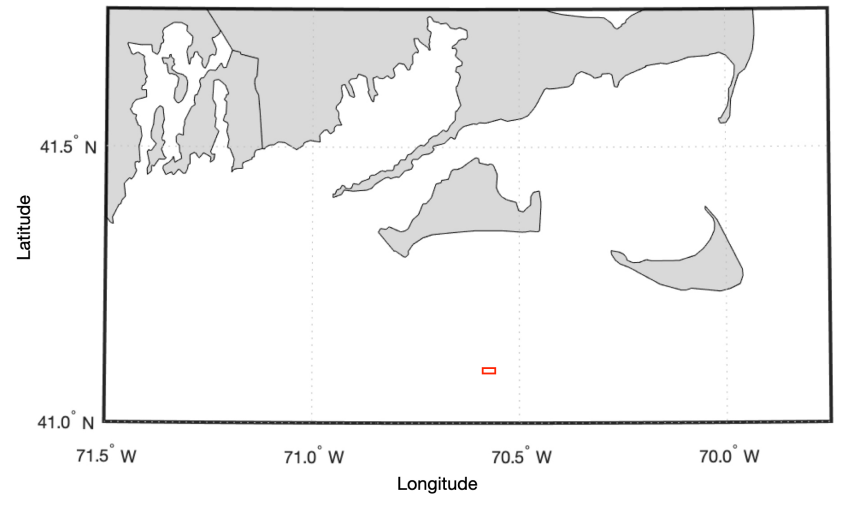

(a)

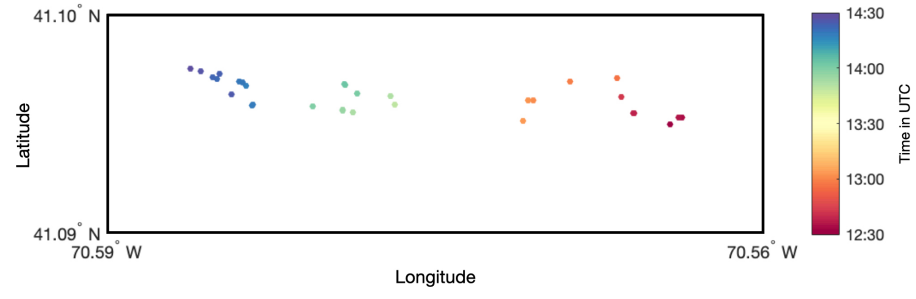

(b)

Figure 1. Location of the in situ samples and photographs. (a) Study site relative to the island of Martha's Vineyard, Massachusetts, USA; (b) Zoom-in on the red rectangle in (a) showing the locations where the drone samples and photographs were taken. The dots are color-coded by time, from red at 12:31 UTC to blue at 14:28 UTC.

Rhodamine WT and ethanol alcohol were mixed to approximate the near-surface seawater density of $1021 \mathrm{~kg} \mathrm{~m}^{-3}$, following the method in [20]. The dye was released from the back of R/V UCONN in an outward spiral pattern, with 3 spiral loops reaching approximately $500 \mathrm{~m}$ in the outermost diameter. The spiral pattern, plotted in Figure 2, started near $40^{\circ} 05.8^{\prime} \mathrm{N}, 70^{\circ} 33.0^{\prime} \mathrm{W}$ at 07:25 EST $/ 11: 25 \mathrm{UTC}$ on 16 August 2018 and finished at 07:58 EST/11:58 UTC. Due to the advection of dye by oceanic currents, the original spiral got distorted over the course of the dye release. About 130 gallons of dye solution containing roughly 20 gallons of pure Rhodamine WT were injected just below the surface using a weight tied to the end of the dye hose. The dye reservoir tank was continually 
stirred to keep the dye solution homogeneous. A movie of the experiment can be found at https: / / youtu.be/0e0fz0F38eg (accessed on 15 October 2021).

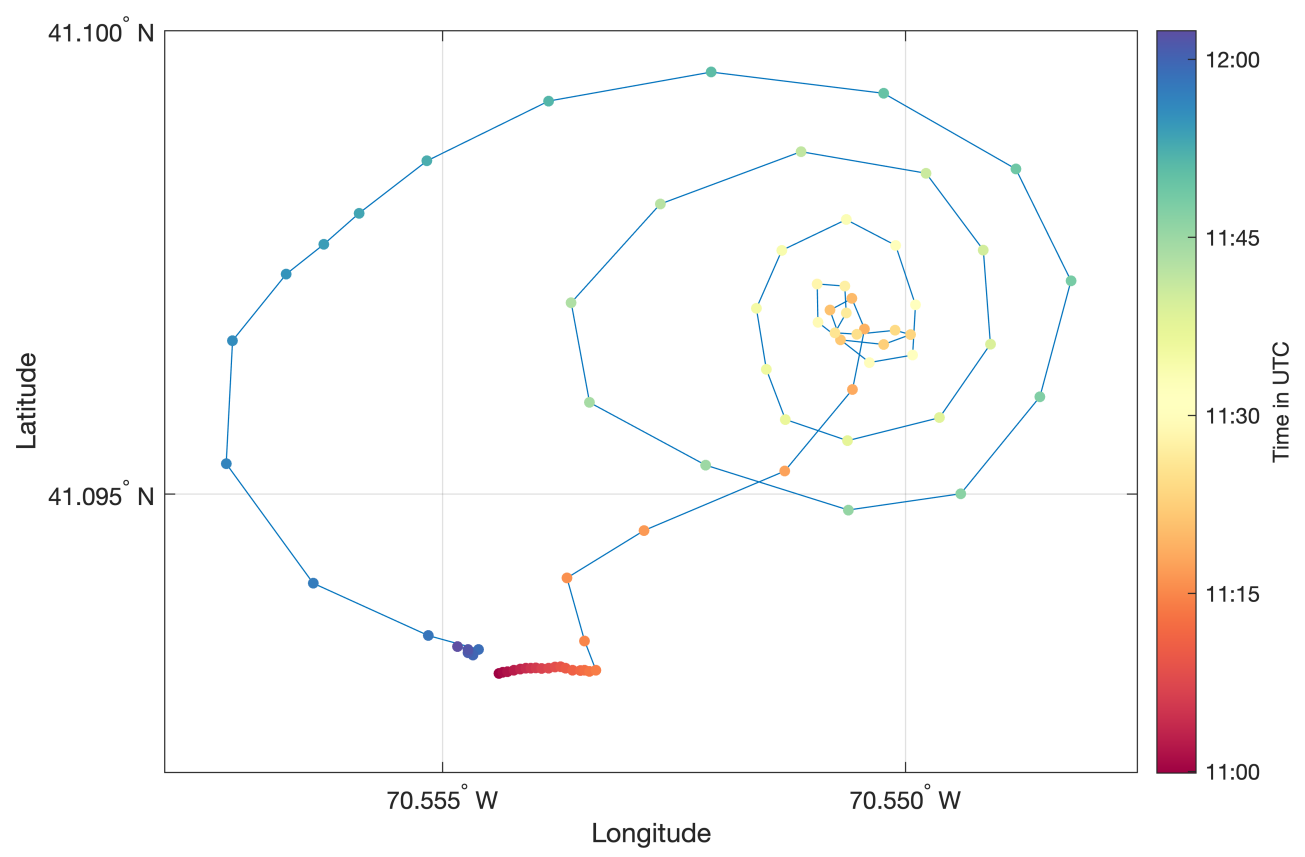

Figure 2. Ship tracks of R/V UCONN showing the spiral pattern of the rhodamine dye release. The dots represent the ship's locations during the release, between 11:25 and 11:58 UTC, and are color-coded by time.

\subsection{DOWSE: DrOne Water Sampling SystEm}

A DrOne Water Sampling SystEm (DOWSE), consisting of a 3D-printed sampling device tethered to a drone, was used to collect water samples at different locations around the dye plume. The water sampler consisted of four separate 3D-printed pieces (.stl files are available here: https:/ / github.com/SchmaleLab/Schmale-Lab-3D-Printing-Files-Bensonet-al-Water-2018, accessed on 15 October 2021) assembled with $2.5 \mathrm{~mm}$ stainless steel screws. The sampler was tethered to the center of a carbon fiber beam on a $4.5 \mathrm{~m}$ orange nylon string tied between two 3D-printed mounts attached to the landing gear. A carabiner was used to quickly attach and remove the water sampler following each collection.

The DOWSE was loaded with a sterile $50 \mathrm{~mL}$ conical tube, and the drone was flown manually to each desired sampling location to collect the samples. Once the drone reached the sampling location, it descended to the surface of the water and allowed the DOWSE to rest on the surface for a few seconds. During this time, the DOWSE filled with water. An overhead image was then taken of the sampling device in the water and $10 \mathrm{~m}$ above the sampling location, which provided a precise record of the location and sampling time embedded in the EXIF file of each of the images. Following each collection, the sample was returned to the boat and the $50 \mathrm{~mL}$ tube (now containing a sample of ocean water with dye) was switched out with a new tube while the drone was still in flight. The drone then flew to another location to collect another sample, until all targeted collections had been completed. The samples were collected over four different flights.

The drone was a Phantom 4 quadcopter (DJI, Shenzhen, China) powered by a $5870 \mathrm{mAh}$ Lithium-ion polymer battery. This battery provided a flight time of about $20 \mathrm{~min}$. The drone weighed $1.38 \mathrm{~kg}$, and was equipped with a gimbaled high definition camera. Additional photography parameters are described in Section 2.4.2. Drone specifications as per the manufacturer can be found at https://www.dji.com/phantom-4/info (accessed on 15 October 2021). 


\subsection{Determination of Rhodamine Dye Concentrations Following Collection with the DOWSE}

Small volume surface collections in the Atlantic Ocean were used to determine Rhodamine dye concentrations following the plume release. Individual locations in close proximity to the ship were sampled by filling $50 \mathrm{~mL}$ conical tubes (Genesee Scientific \#28-108) with ocean water. We utilized the DOWSE sampling system to retrieve the ocean water samples [15]. Samples were stored on board in the dark.

A Turner Designs handheld data bank (serial \#2900495) was used on board to determine the Rhodamine concentration of each sample. The Rhodamine sensor (serial \#2104382) was calibrated on board using a solid-state calibration unit (P/N 2100-900) set to $20 \mathrm{ppb}$. Immediately following calibration, the Rhodamine sensor was placed in a $50 \mathrm{~mL}$ conical tube with $15 \mathrm{~mL}$ of the collected ocean sample. The sensor was held in place $2.4 \mathrm{~cm}$ from the bottom of the tube and concentration values were logged in the dark. The concentration of Rhodamine in each drone water sample was recorded in triplicate on the same afternoon.

The dye concentrations at each of the sampling points are shown in Figure 3.

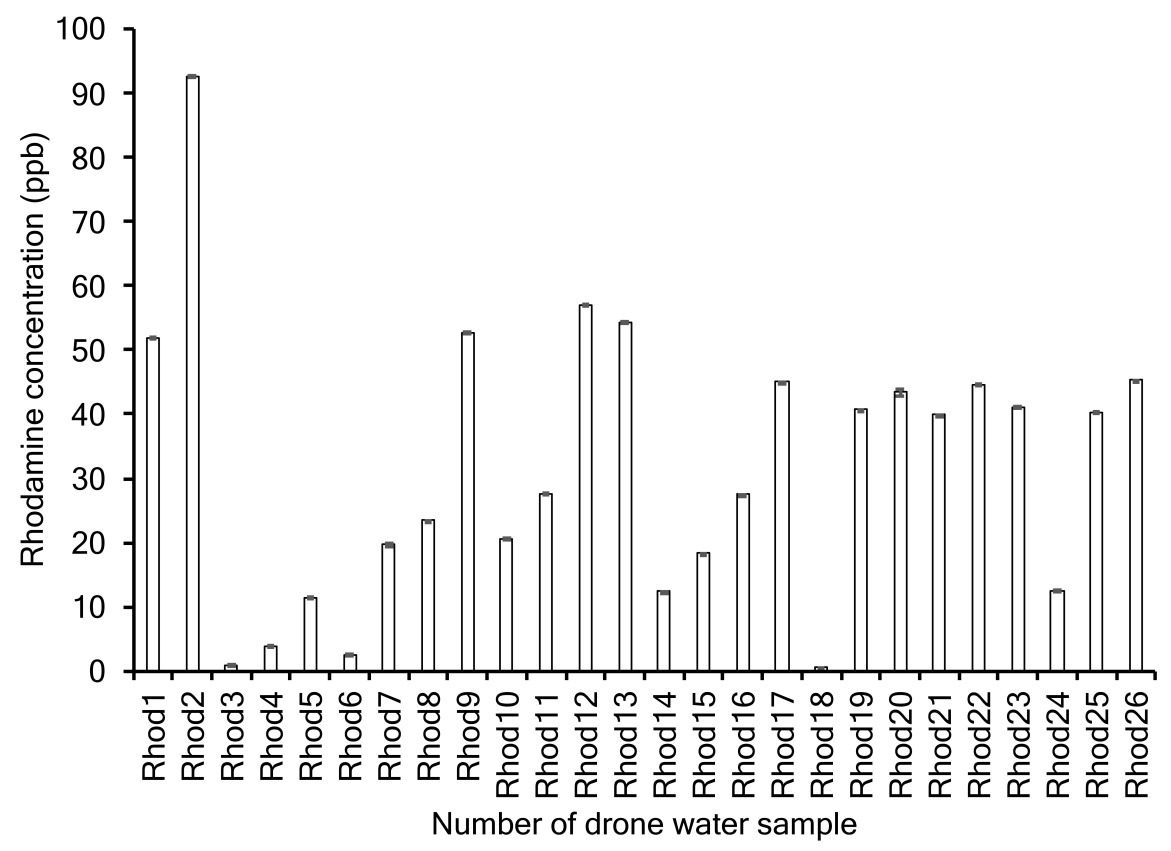

Figure 3. Mean concentrations of rhodamine dye from each of the water samples (Rhod1 through Rhod26) collected with the DrOne Water Sampling SystEm (DOWSE). The measurements were made with a fluorometer onboard the research vessel Connecticut immediately after they were collected from the ocean. Error bars represent the standard error of the mean of three independent concentration measurements for each sample.

\subsection{Photogrammetry}

The DJI Phantom 4 quadcopter's gimbaled camera recorded images at each dye sampling location, typically capturing an image close to the surface and an image at about $10 \mathrm{~m}$ of altitude. Out of 26 sampling locations, 50 images were captured, with locations 5 and 9 not having surface images. The average lag between the surface and the altitude images was $14.3 \mathrm{~s}$ and the average difference in the gimbal's yaw was $5.6^{\circ}$. To compare the distribution of dye between the surface images and the altitude images, the sea surface footprint (i.e., the extent of sea surface photographed in each image) had to be calculated from the gimbal's rotation and altitude.

To calculate the altitude and the sea surface footprint, the parameters used were as follows: the camera was a $1 / 2.3^{\prime \prime}$ CMOS with a sensor size of 6.17 -by- $4.55 \mathrm{~mm}$. The lens had a $20 \mathrm{~mm} \mathrm{f} / 2.8$ focus at $\infty$ and a focal length of $3.61 \mathrm{~mm}$. The field of view parameters were 
$F O V_{h}=94.4^{\circ}$ in the horizontal and $F O V_{v}=61.9^{\circ}$ in the vertical. The image dimensions were 4000-by-2500 pixels.

\subsubsection{Image Pre-Processing and Altitude Calculations}

For each image, the gimbal's orientation (roll, pitch and yaw) was used to compute the corresponding rotation matrix $R$ and correct the image's distortion. For all images, there was no roll angle. On average, the pitch angle was $0.9^{\circ}$. The latitude and longitude coordinates of each image were converted to Easting and Northing in UTM Zone 19 N.

The drone flight logs recorded the following altitude measurements, all in meters: the OSD altitude, which is the altitude at which the picture was taken; the Home height, which is the reference height used for calibration; and the OSD height, which yields the height of the picture above ground. The difference between OSD altitude and Home height should have given the altitude of the image, but upon inspection, this difference did not explain the OSD height and often resulted in negative altitudes, even for pictures that had clearly been taken above sea level.

The drone's altitude measurements being prone to errors, the altitude of each image was calculated using a photographed object as a reference: typically, a drifter or the DOWSE sampler floating at the surface. The sampler measured $\sim 7.75$ in or $196.85 \mathrm{~mm}$ in length and $70 \mathrm{~mm}$ in diameter, and the drifter sails measured $\sim 1.09 \mathrm{~m}$. Out of 26 images taken at altitude, only 4 images had captured an object of reference at the surface: images 2 and 5, which captured a drifter, image 16, which captured the vessel's auxiliary Rigid-hull Inflatable Boat (RIB), and image 22, which captured floating algae. Image 5 did not have a surface equivalent.

The altitude is given by:

$$
\text { altitude }=\text { Focal Length } \times \frac{\text { measured length of the object }}{\text { distance on camera }}
$$

The distance on camera, or length of object, is the length of the object of interest in pixels over the focal plane resolution:

$$
\text { distance on camera }=\frac{\text { length of object in pixels }}{\text { Focal Plane Resolution }}
$$

The focal plane resolution in pixel per $\mathrm{mm}$ was obtained through the following ratio:

$$
\text { Focal Plane Resolution }=\frac{\text { Pixel Dimension }}{\text { Sensor size }}
$$

The reference object and resulting altitude for each image are listed in Table S1 of the Supplementary Materials.

\subsubsection{Sea Surface Footprint}

The sea surface footprint was calculated from the gimbal's rotation $\mathbf{R}$, the UTM coordinates in Zone $19 \mathrm{~N}$ of each image and the camera specifications. From the FOV, the rays delimiting the boundaries of what is captured by the camera can be calculated as follows:

$$
\left[\begin{array}{lll}
+\tan \left(F O V_{h} / 2\right) & +\tan \left(F O V_{v} / 2\right) & -1 \\
+\tan \left(F O V_{h} / 2\right) & -\tan \left(F O V_{v} / 2\right) & -1 \\
-\tan \left(F O V_{h} / 2\right) & -\tan \left(F O V_{v} / 2\right) & -1 \\
-\tan \left(F O V_{h} / 2\right) & +\tan \left(F O V_{v} / 2\right) & -1
\end{array}\right]
$$

The rays were then rotated according to $\mathbf{R}$ and their intersections with the sea surface were calculated, assuming an altitude of $0 \mathrm{~m}$ for the surface. The extent of sea surface coverage resulted from the difference of these intersection points. 


\subsubsection{Dye Heat Maps}

All images were imported in MATLAB and decomposed into their red, green and blue (RGB) components, ranging from 0 to 255 . The percentage of the red component was calculated for the pre-processed image, which was rotated and slightly distorted from the original due to the gimbal's rotation. An example is provided for Rhod 5, the image at sample location \#5, shown in Figure 4a. The resulting heat map of red ratio is shown in Figure $4 \mathrm{~b}$. While the wave crests were still discernible in the heat map, the signature of the sun rays, which were still visible in the RGB decomposition, have been erased in the heat map. The limits of the dye plume are discernible from the difference of red ratio. The buoys on the drifters, which were white and have no rhodamine, had lower ratios of red, around $\frac{1}{3}$.

To construct the heat maps of the dye distribution, the assumptions were as follows: first, the rhodamine's signature on the images corresponds to a high ratio of red; second, the ambient seawater's signature on the images corresponds to a ratio of red approaching zero due to the ocean light absorption.

\subsubsection{Dye Calibration}

The constructed heat maps were then calibrated using the mean rhodamine concentrations in $\mathrm{ppb}$, which have been sampled in situ, as explained in Section 2.3. The assumptions were as follows. First, the in situ samples, $p p b_{\text {high }}$, were taken at the centers of the images: for calibration, the red ratio ratio $_{\text {high }}$ taken at the center of the image corresponds to the in situ concentration in $\mathrm{ppb}$. Second, the ambient seawater has no red component, hence $0 \mathrm{ppb}$ corresponds to a red null ratio. The second assumption was verified against images that captured patches of blue ocean: the red ratios were 0 or close to 0 . An example is shown in Figure 4c for the picture in Figure 4a. The in situ rhodamine sample had a concentration of $11 \mathrm{ppb}$, the center of the image had a red ratio of 0.4806 and the lowest ratio in the picture was 0.2827 . The patches of blue ocean are even more contrasted than in the ratio ratio heat maps. The limits of the dye plume, the drifter and the DOWSE are more easily discernible.

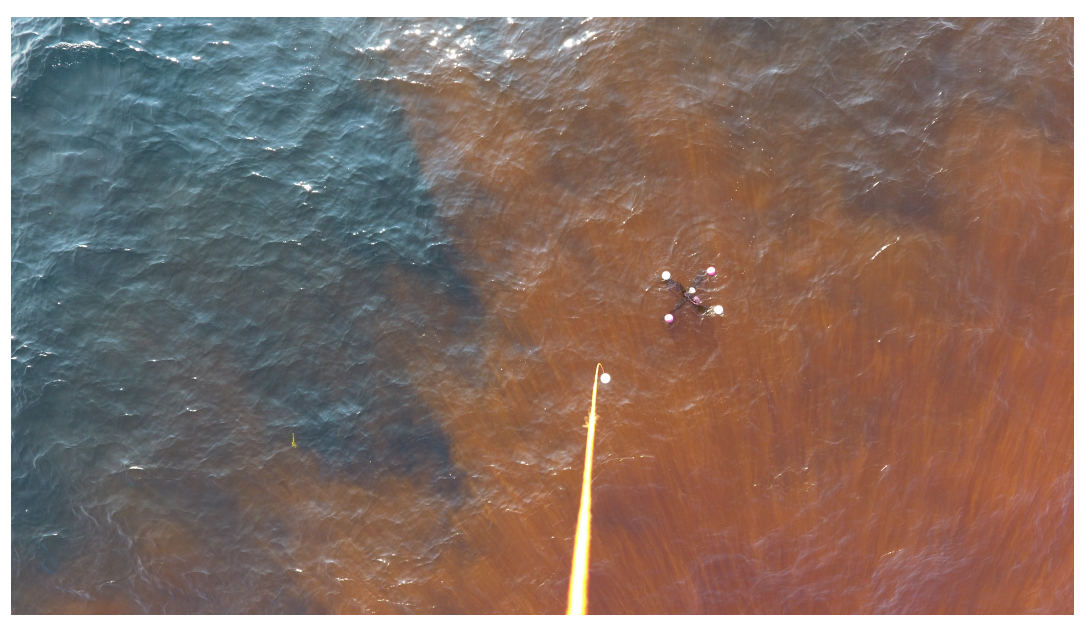

(a)

Figure 4. Cont. 


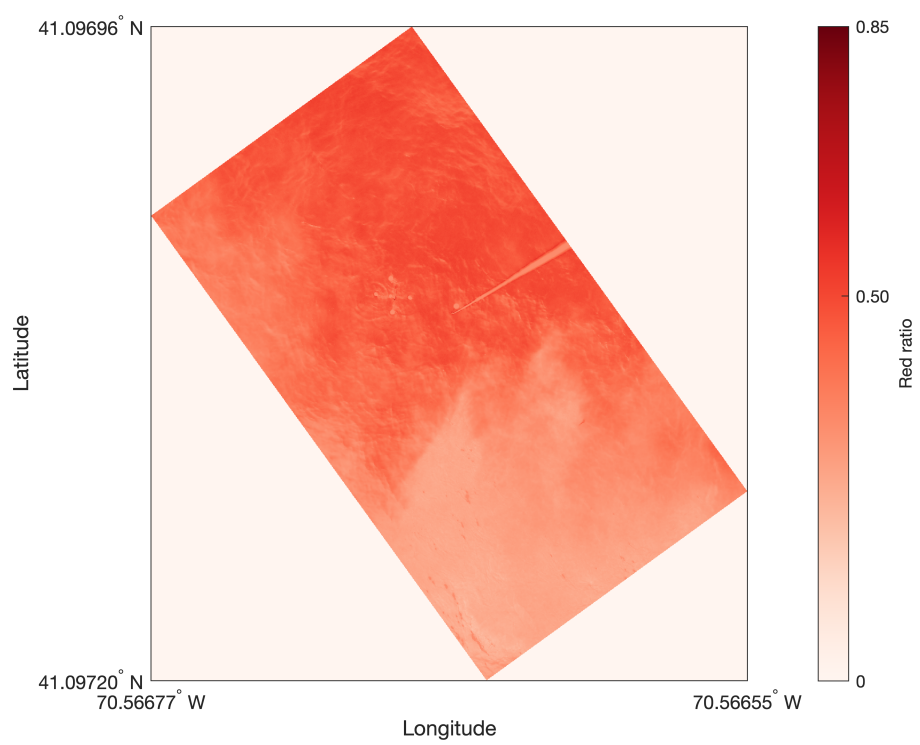

(b)

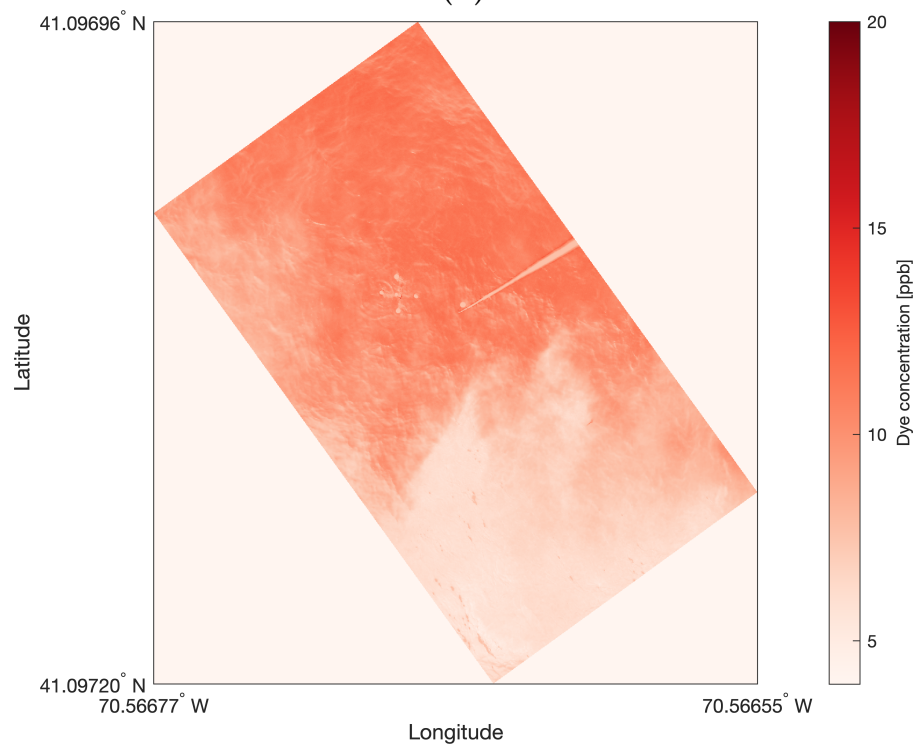

(c)

Figure 4. Color decomposition for the altitude image at sample location \#5. (a) Raw image. For scale, the drifter had a span of $1.09 \mathrm{~m}$; (b) Heat map of the red ratios: the percentage of red component over total RGB intensities; (c) Heat map of dye concentrations calibrated with the in situ samples.

\section{Results}

\subsection{Dye Distribution}

The potential association between red ratios and dye concentration in ppb was investigated for the surface images. For each image, the mean red ratios were calculated and plotted against the in situ measurements in ppb, as shown in Figure 5 (Top). A linear regression was fit from $\left[0\right.$, ratio $\left._{\text {mean }}\right]$ to the two datapoints $\left[0, p p b_{\text {high }}\right]$ using MATLAB's embedded polyfit.m function and then evaluated at all red ratio datapoints. The resulting $R^{2}$ value was 0.511 for a linear fit. The datapoints were color-coded in red for the images saturated with dye and in blue for the images that contained some blue ocean. For the latter group, the distribution was more scattered, and the $R^{2}$ value of the linear fit increases to 0.566 when excluding the images with patches of blue ocean and focusing on images saturated with rhodamine. 

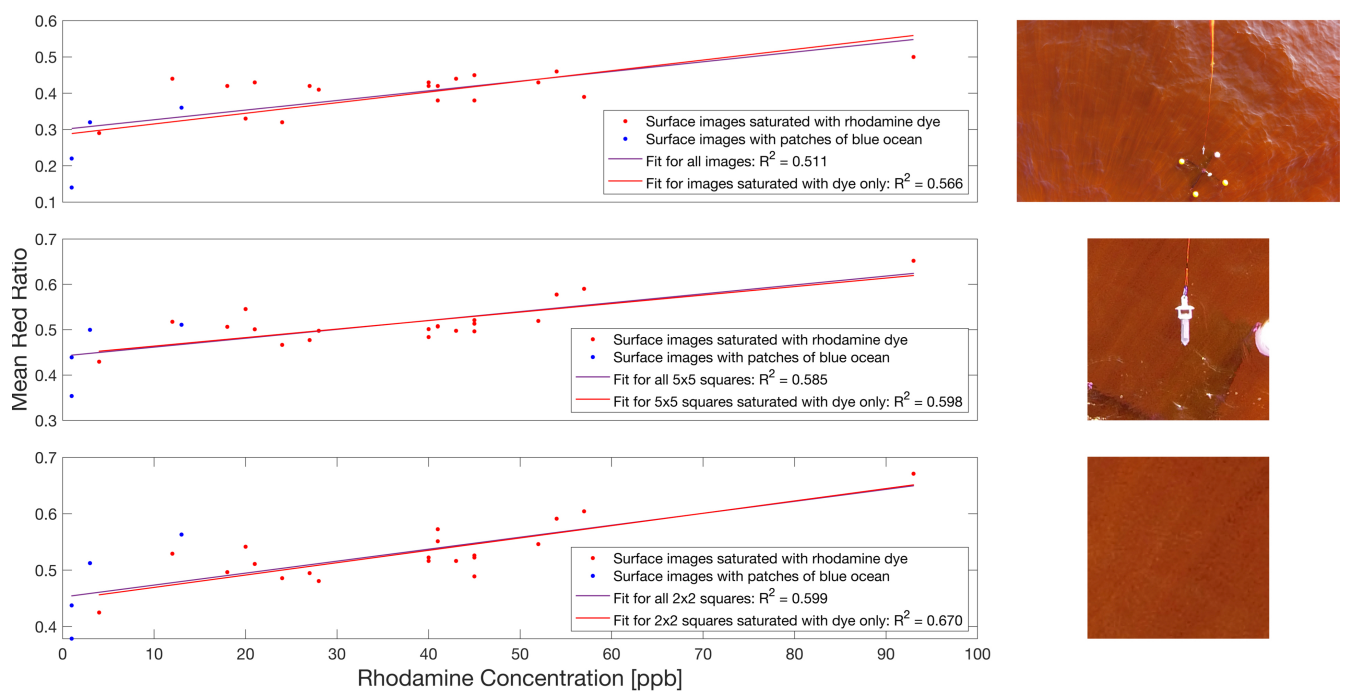

(a)

(b)

Figure 5. (a) Correlation between average high/low red ratios and in situ measurements of dye in $\mathrm{ppb}$ for the surface images. Images that contained blue ocean were color-coded in blue. Images that were saturated with dye are color-coded in red. (Top) Correlation for the entire surface images. (Middle) Correlation for the $5 \times 5$-in squares around the sampler in the surface images. (Bottom) Correlation for the $2 \times 2$-in squares within the $5 \times 5$-in squares. (b) Example images for Rhod 2 .

Given that the images were photographed within three hours of the dye release, and given that the dye diffusion is not homogeneous (see [20,25] for additional patterns of dye diffusion in the surrounding coastal ocean), the analysis was re-iterated on subsets of the surface images to look at the correlation between in situ dye concentrations and averaged red ratios over smaller areas. For these additional analyses, the surface images were cropped around their center to capture about 5 -in squares of sea surface, then cropped to 2 -in squares within these 5 -in squares but without capturing the sampler itself. The results are plotted in Figure 5, (Middle) and (Bottom). In both cases, the linear fit was calculated for the set of all images and for the set of images at saturation (no patches of blue ocean). The $R^{2}$ values increased to 0.598 for the saturated $5 \times 5$-in squares and to 0.670 for the saturated $2 \times 2$-in squares.

\subsection{Comparisons between Surface and Altitude Images}

For the 24 sample locations where both surface and altitude images were captured, the distributions of calibrated dye data, in ppb, were plotted as histograms. To quantify the similarity between the surface and altitude histograms, the histogram intersection [26] was used as a metric, with the intersection values being in the range from 0 for no overlap to 1 for identical distributions. Figure 6 presents six examples: in the top panel, one at a location with low rhodamine concentrations (\#4), a location with medium concentrations (\#11) and a location with high concentrations (\#1). The bottom panel presents the histograms for the 3 locations where both surface and altitude images had an altitude reference.

In most of the 24 histogram pairs, the surface and the altitude images exhibited similar distributions, with the histograms overlapping in most cases. The average value for histogram intersection was 0.469 and the highest amount of overlap was found at location \#19 where the intersection value was 0.830 . Three pairs, locations \#12, \#16 and \#23, showed very little to no overlap, as illustrated in Figure 6e for \#16. For pair \#12, the time difference was $15.0 \mathrm{~s}$ and the yaw difference was $10^{\circ}$; both differences were slightly higher than average and the intersection value was 0.038 . For pair \#16, the time difference was $33.0 \mathrm{~s}$ and the yaw difference was $0.05^{\circ}$ only, but the RIB was present in the altitude imageand the altitude image was taken at $20.78 \mathrm{~m}$, over $16.5 \mathrm{~m}$ higher than the surface image. The histograms for this location had the lowest intersection value at $2.53 \times 10^{-5}$. 
For pair \#23, the time difference was $10.0 \mathrm{~s}$, the yaw difference was $0.1^{\circ}$ and the intersection value was 0.070 . Overall, the altitude histograms aligned quite well with the surface histogram, meaning that qualitatively, the calibrated dye distributions as measured by the altitude images were a good proxy for the calibrated dye distributions as measured by the surface images.

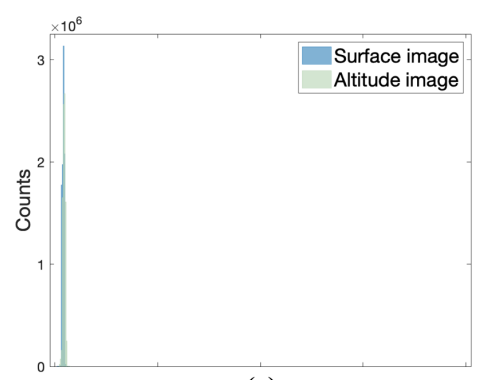

(a)



(d)

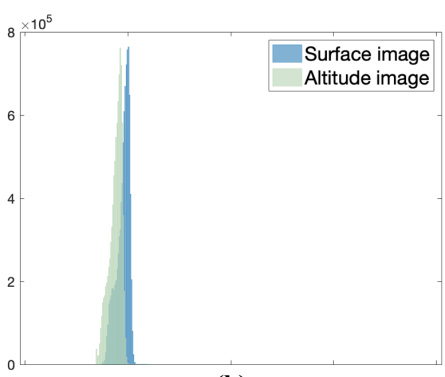

(b)

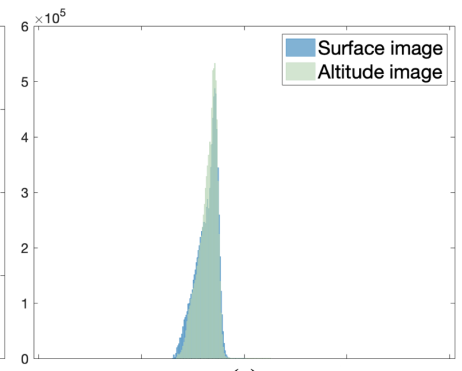

(c)

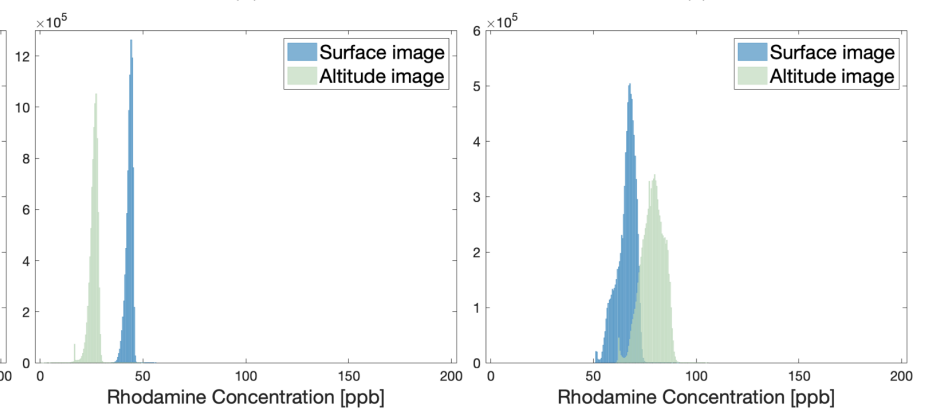

(e)

(f)

Figure 6. Distributions of calibrated heat maps for selected pairs of surface (blue) and altitude (green) images. The bin widths were $0.5 \mathrm{ppb}$ for all histograms. Top row: locations (a) \#4, (b) \#11 and (c) \#1 had distributions that overlapped quite well, with low, medium and high rhodamine concentrations, respectively. The similarity values were $0.584,0.450$ and 0.823 , respectively. Bottom row: distributions for the three pairs that had an object of reference at altitude, locations (d) \#2, with a similarity value of 0.432 , (e) \#16, with a similarity value of $2.53 \times 10^{-5}$ and (f) \#22, with a similarity value of 0.181 .

As described in Table S1, three pairs of images had an object of reference at altitude: locations \#2, \#16 and \#22, which used a surface drifter, the RIB and a piece of floating algae for reference, respectively. The absolute differences are presented in the histograms. For each pair, the calibrated dye heat map at altitude was interpolated onto the grid of the calibrated dye heat mat at the surface. The percent difference between dye concentrations in $\mathrm{ppb}$ were calculated and the results are shown for the first pair, \#2, in Figure 7c. The results for pair \#16 and pair \#22 are shown in the Supplementary Materials in Figures S1 and S2, respectively. For location \#2, the distributions from the surface and altitude images were quite similar, except where the wave crest and the drifter were present in Figure 7a,b: $52.07 \%$ of the datapoints were within $\pm 10 \%$ and $78.49 \%$ of the datapoints were within $\pm 20 \%$, with an average percent difference between the distributions of $15.15 \%$. For location \#16, in spite of the wave crests in the surface image (Figure S1a) and the presence of the RIB in the altitude image (Figure S1b), the distribution from the altitude image was quite similar to the distribution from the surface image, with an average percent difference of $8.55 \%$. $68.31 \%$ of the datapoints were within $\pm 10 \%$ and $92.04 \%$ of the datapoints were within $\pm 20 \%$. For location \#22, $21.20 \%$ of the datapoints were within $\pm 10 \%, 52.08 \%$ of the datapoints were within $\pm 20 \%$ and the average percent difference was $21.59 \%$. Once again, the wave crests in the surface image (bottom of Figure S2a) were discernible in the heat map in Figure S2c. 


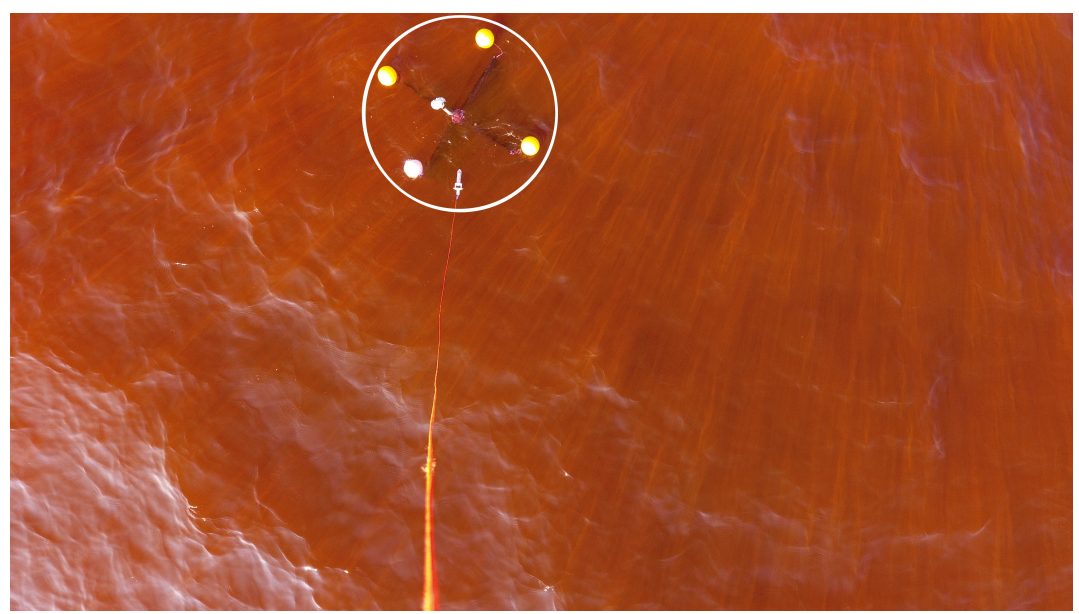

(a)

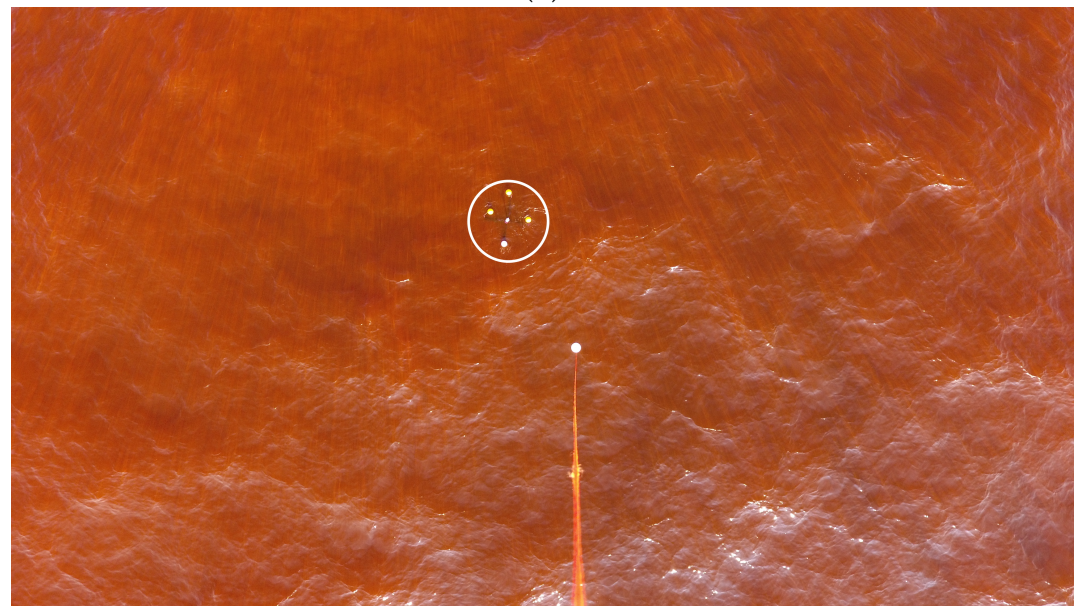

(b)

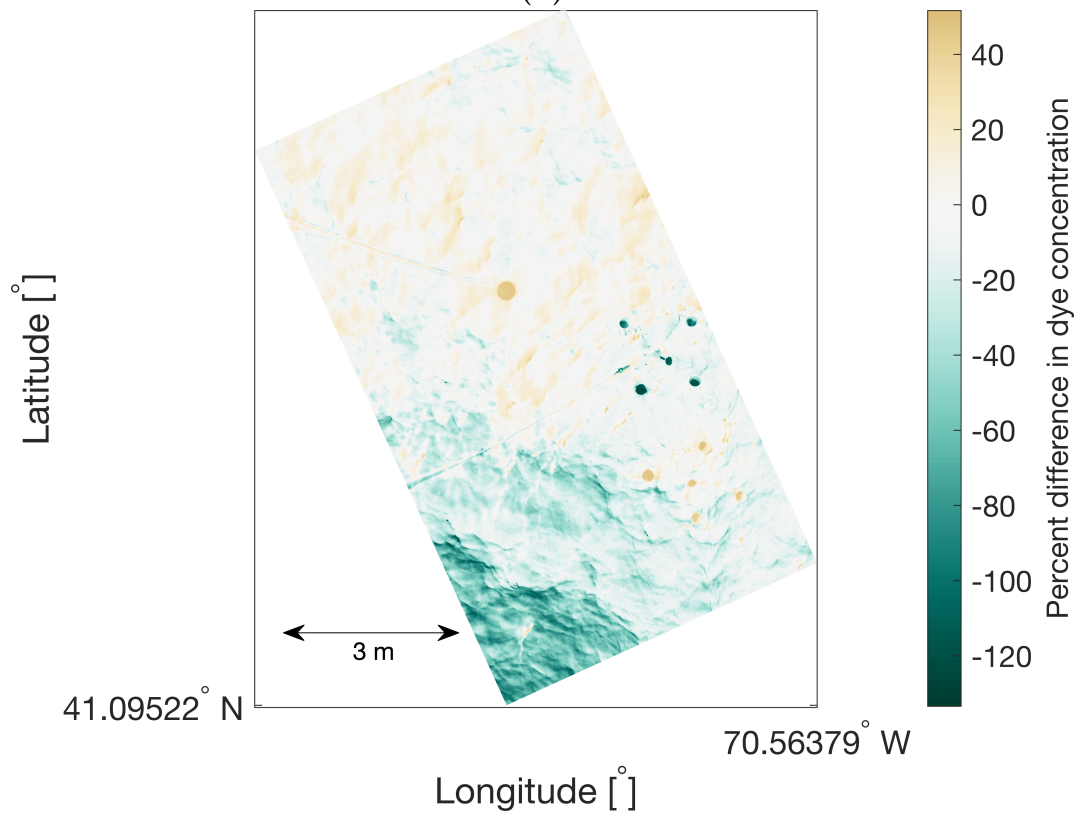

(c)

Figure 7. Comparison of dye heat maps between the surface and altitude images at sample location \#2; (a) Raw surface image. The 1.09-m drifter, circled in white, was used as altitude reference; (b) Raw altitude image. The 1.09-m drifter, circled in white, was used as altitude reference; (c) Percent difference in rhodamine concentration in ppb between the photographs in $(\mathbf{a}, \mathbf{b})$. 
The signatures of the drifters and the sampler were not removed and appeared sharply in the heat maps, as expected. With a small dataset of 50 images, no machine learning object detection method could have been easily implemented, as such algorithms require much greater datasets to be trained. Removing the objects would have had to be done manually, which is very time consuming.

\section{Discussion}

Rhodamine dye (a surrogate hazardous agent) was released into the Atlantic ocean in August, 2018, and a series of experiments were conducted to track the dye near the water surface within three hours following the release. Our observed range of dye concentrations (1 ppb to $93 \mathrm{ppb}$ ) was similar to dye concentrations commonly used in recent scientific studies; Powers et al. [5] released fluorescein into a freshwater lake and estimated concentrations using a quadcopter, and Rypina et al. [20] released a mixture of rhodamine and fluorescein into the ocean and concentrations were measured with a shipboard flowthrough fluorometer.

The red ratio, or the percentage of the red component within the RGB decomposition, was calculated as a proxy to construct heat maps of dye within each picture. Though the red ratio filtered out the signature of the sun rays, there were a number of limitations to this approach as a predictor of dye concentrations. First, the red ratio was unable to reduce the signature of the wave action; consequently wave crests are still discernible in the heat maps. Future dye transport studies could be conducted in calm bays or inlets to eliminate wave crest caveats (e.g., Powers et al. [5]). Second, glint (momentary flashes of light from the sun's reflection on the water surface), was observed at the edges of some of the raw images, and the red ratio was unable to account for these 'glinted areas'. Future missions might consider the use of a proper polarizing camera filter, as such filters have been shown to limit this nuisance in images of the water surface (e.g., Shaw and Vollmer [27]). Third, the red ratio does not incorporate a working knowledge of the vertical dye plume and/or any potential stratification beneath the sea surface (e.g., Lee et al. [28] who looked into models of vertical profiles of Chlorophyll from remote sensing of ocean color). Future missions could incorporate sub-surface sampling of the dye using different types of dronebased water sampling devices (e.g., Castendyk et al. [29]) coupled with multiple Secchi disk observations (e.g., Lee et al. [30]) at the sampling locations, but these additional observations were beyond the scope of resources and capabilities available to the present study. Consequently, dye estimates based on red ratios derived from our drone images may in fact include some dye beneath the water surface. Fourth, shadows from clouds observed in some of the images (e.g., Li et al. [31], Zhang et al. [32]) and those from the drone and the sampling tether line were not taken into account in this analysis. Measuring the self-shadow effect of marine instruments is an active area of research (see Lin et al. [33] and references therein) and these effects could also have contributed to small errors in dye estimates using the red ratio approach.

Despite these limitations, heat maps of rhodamine concentrations (ppb) constructed by calibrating the red ratios with the in situ concentrations revealed linear fits $\left(R^{2}\right)$ ranging from 0.511 to 0.670 . The linear fit was constructed using two points of reference for dye concentration, assuming that the mean red ratio in the images or their subset corresponded to the measured concentrations in $\mathrm{ppb}$ and that a red ratio of 0 corresponded to $0 \mathrm{ppb}$. A major uncertainty in this approach is the assumption that each image's center corresponded to the sample of water collected by DOWSE: the images having been taken after the sample collection, we were unable to measure the red ratio of the exact parcel of water sampled. It is assumed that for a parcel of water small enough, i.e., the volume of water around the collected sample, the dye distribution was homogeneous. The $R^{2}$ values increased from 0.566 to 0.598 to 0.670 as the saturated images were cropped to $5 \times 5$-in and to $2 \times 2$-in squares, which is in agreement with the assumption that the pixels near the center of the images correspond to the parcel of water that was sampled. The red ratios calculated from aerial photographs thus appear to be good indicators of the rhodamine concentration at 
the surface. The limitations of this assumption are however reflected in the correlation plots in Figure 5: for the images that had captured some blue ocean, the red ratios were more scattered, most likely because the dye distribution was much more heterogeneous. The assumption that a zero red ratio corresponded to $0 \mathrm{ppb}$, however, was verified from the blue ocean (no dye) pictures.

Quantitative comparisons of the dye spatial coverage were possible at three locations that had altitude references for both images. At location \#2, the images were taken at an altitude difference of $11.1 \mathrm{~m}$ and the average percent difference between dye concentrations was $15.15 \%$ (see Figure 7c). At location \#22, the altitude difference was $10.3 \mathrm{~m}$ and the average percent difference was $21.59 \%$. At location \#16, the altitude difference was $16.5 \mathrm{~m}$ yet the heat maps differed much less: the average percent difference was $8.55 \%$. The relatively low percent differences are consistent with Zeng et al. [17], who found "negligible" differences in water spectra from images taken at a $100 \mathrm{~m}$ altitude compared to the spectra from images taken at a $20 \mathrm{~m}$ altitude. It is important to remember that the estimates of dye concentrations and the comparison between surface and altitude estimates were calculated to determine the loss of accuracy with altitude. The initial plan in the experimental design was to capture the presence of a surface drifter in each image and to sample dye adjacent to the drifter. The field conditions presented some challenges, however, and the fast currents led to a rapid dye dispersion. Consequently, only a few sets of images of higher altitude had objects of reference. In future studies, an object of reference will be dropped at each sample location to help with the altitude calibration.

The ability to obtain quantitative results was limited in part by the lack of accurate altitude measurements. A high-accuracy altimeter on the drone's gimbal would have enabled the calculations of photometric footprints for all images and allowed for a more systematic comparison. Removing the DOWSE sampler and other objects captured within the images would also yield greater results, but doing so manually is very time-intensive and the sample size was way too small for an automatic approach using machine learning detection algorithms. As more studies are conducted with DOWSE and more images are obtained, the removal of such objects can be automated. The main source of error in the resulting heat maps would then come from the wave action: indeed, the RGB filtering was not sufficient to remove the wave crests. Note that this study was conducted on a very sunny day and it is unclear how much the weather, beyond the waves, would affect the results. It would be of interest to investigate the role of different environmental factors, similarly to Zeng et al. [17]. The present study was designed to monitor dye around a single dye release within a period of about three hours. Consequently, it is not possible to extend the results to a range of atmospheric and hydrological conditions, but there is an opportunity for future studies to target multiple dye releases under a range of wave regime, cloud coverage or water quality.

Coordinated manned and unmanned systems may be used to detect, track and assist in mitigating hazardous agents in the future. In this study, the surface and altitude drone images were sufficient to detect the dye plume. The concentration estimates from the altitude images were roughly similar to the surface estimates, meaning that if high accuracy is not needed when tracking a plume, drone flights can be reduced by staying at an altitude of about $10 \mathrm{~m}$. This would save survey time, battery life, and would reduce the risk of potential equipment failure due to waves splashing on the drone. As a final remark, this study focused on surface dynamics and did not consider three-dimensional effects nor vertical transport. Many hazardous agents stay at the surface due to buoyancy constraints: with a density of $858 \mathrm{~kg} \mathrm{~m}^{-3}$ [34], the Deepwater Horizon oil was buoyant and many oil particles rose to the surface, for instance. A surface study is thus still relevant to help track such hazards.

Supplementary Materials: The following are available online at https:/ / www.mdpi.com/article/ $10.3390 / \mathrm{rs} 13214415 / \mathrm{s} 1$. Table S1. Objects of reference and their measured lengths for the altitude calculations. Figure S1. Comparison of dye heat maps between the surface and altitude images at sample location \#16. (a) Raw surface image. The 7-cm sampler, circled in white, was used as 
altitude reference. (b) Raw altitude image. The 14-foot RIB, circled in white, was used as altitude reference. (c) Percent difference in rhodamine concentration in ppb between the photographs in $(a, b)$. Figure S2. Comparison of dye heat maps between the surface and altitude images at sample location \#22. (a) Raw surface image. The 7-cm sampler, circled in white, was used as altitude reference. The white arrow indicates the algae used in (b) raw altitude image. The algae from (a), circled in white, was used as altitude reference. (c) Percent difference in rhodamine concentration in ppb between the photographs in $(a, b)$.

Author Contributions: M.F. participated in field experiment, analyzed data and led the writing of the manuscript. R.H. designed and conducted experiments, analyzed data and assisted in writing the manuscript. D.G.S.III managed the project, designed experiments, operated the UAS, analyzed data and assisted in writing the manuscript. I.I.R. and B.A.H. coordinated the dye release. T.P. coordinated the field experiments. All authors have read and agreed to the published version of the manuscript.

Funding: This research was supported in part by the U.S. National Science Foundation (NSF) under Grant Numbers AGS 1520825 (Hazards SEES: Advanced Lagrangian Methods for Prediction, Mitigation and Response to Environmental Flow Hazards), and IIS-1637915 (NRI: Coordinated Detection and Tracking of Hazardous Agents with Aerial and Aquatic Robots to Inform Emergency Responders). Any opinions findings, and conclusions or recommendations expressed in this material are those of the authors and do not necessarily reflect the views of the NSF. The authors thank the crew of R/V UCONN for their help and assistance during the field experiments.

Institutional Review Board Statement: Not applicable.

Informed Consent Statement: Not applicable.

Data Availability Statement: Data can be found at https:/ / github.com/NSF-ALPHA/Dye_Tracking_ with_Drones, (accessed on 15 October 2021)

Acknowledgments: The authors thank the crew aboard R/V Connecticut for their support and assistance during the field operations.

Conflicts of Interest: The authors declare no conflict of interest.

Abbreviations
The following abbreviations are used in this
DOWSE

\section{References}

1. Ji, C.; Beegle-Krause, C.J.; Englehardt, J.D. Formation, detection, and modeling of submerged oil: A review. J. Mar. Sci. Eng. 2020, 8, 642. [CrossRef]

2. Ore, J.P.; Elbaum, S.; Burgin, A.; Detweiler, C. Autonomous aerial water sampling. J. Field Robot. 2015, 32, 1095-1113. [CrossRef]

3. Nolan, P.J.; Pinto, J.; González-Rocha, J.; Jensen, A.; Vezzi, C.N.; Bailey, S.C.C.; de Boer, G.; Diehl, C.; Laurence, R., III; Powers, C.W.P.; et al. Coordinated Unmanned Aircraft System (UAS) and Ground-based Weather Measurements to Predict Lagrangian Coherent Structures (LCSs). Sensors 2018, 18, 4448. [CrossRef] [PubMed]

4. Barbieri, L.; Kral, S.T.; Bailey, S.C.C.; Frazier, A.E.; Jacob, J.D.; Reuder, J.; Brus, D.; Chilson, P.B.; Crick, C.; Detweiler, C.; et al. Small Intercomparison of Small Unmanned Aircraft System (sUAS) Measurements for Atmospheric Science during the LAPSE-RATE Campaign. Sensors 2019, 19, 2179. [CrossRef] [PubMed]

5. Powers, C.; Hanlon, R.; Schmale, D.G. Tracking of a Fluorescent Dye in a Freshwater Lake with an Unmanned Surface Vehicle and an Unmanned Aircraft System. Remote Sens. 2018, 10, 81. [CrossRef]

6. Wu, D.; Li, R.; Zhang, F.; Liu, J. A review on drone-based harmful algae blooms monitoring. Environ. Monit. Assess. 2019, 191, 211. [CrossRef]

7. Dugan, J.P.; Piotrowski, C.C.; Williams, J.Z. Rapid environmental assessment of nearshore METOC fields using motion imaging techniques applied to surrogate UAV data. Ocean. Conf. Rec. 2002, 4, 1956-1961. 
8. Lomax, A.S.; Corso, W.; Etro, J.F. Employing unmanned aerial vehicles (UAVs) as an element of the Integrated Ocean Observing System. In Proceedings of the OCEANS 2005 MTS/IEEE, Washington, DC, USA, 17-23 September 2005; Volume 1, pp. 184-190.

9. Casella, E.; Collin, A.; Harris, D.; Ferse, S.; Bejarano, S.; Parravicini, V.; Hench, J.L.; Rovere, A. Mapping coral reefs using consumer-grade drones and structure from motion photogrammetry techniques. Coral Reefs 2017, 36, 269-275. [CrossRef]

10. Tauro, F.; Porfiri, M.; Grimaldi, S. Surface flow measurements from drones. J. Hydrol. 2016, 540, 240-245. [CrossRef]

11. Detert, M.; Weitbrecht, V. A low-cost airborne velocimetry system: Proof of concept. J. Hydraul. Res. 2015, 53, 532-539. [CrossRef]

12. Bandini, F.; Butts, M.; Jacobsen, T.V.; Bauer-Gottwein, P. Water level observations from unmanned aerial vehicles for improving estimates of surface water-Groundwater interaction. Hydrol. Process. 2017, 31, 4371-4383. [CrossRef]

13. Bandini, F.; Jakobsen, J.; Olesen, D.; Reyna-Gutierrez, J.A.; Bauer-Gottwein, P. Measuring water level in rivers and lakes from lightweight Unmanned Aerial Vehicles. J. Hydrol. 2017, 548, 237-250. [CrossRef]

14. Schmale, D.G.; Ross, S.D.; Fetters, T.L.; Tallapragada, P.; Wood-Jones, A.K.; Dingus, B. Isolates of Fusarium graminearum collected 40-320 m above ground level cause Fusarium head blight in wheat and produce trichothecene mycotoxins. Aerobiologia 2012, 28, 1-11. [CrossRef]

15. Benson, J.; Hanlon, R.; Seifried, T.M.; Baloh, P.; Powers, C.W.; Grothe, H.; Schmale, D.G. Microorganisms Collected from the Surface of Freshwater Lakes Using a Drone Water Sampling System (DOWSE). Water 2019, 11, 157. [CrossRef]

16. Partama, I.G.Y.; Kanno, A.; Ueda, M.; Akamatsu, Y.; Inui, R.; Sekine, M.; Yamamoto, K.; Imai, T.; Takaya Higuchi, T. Removal of water-surface reflection effects with a temporal minimum filter for UAV-based shallow-water photogrammetry. Earth Surf. Process. Landforms 2018, 43, 2673-2682 [CrossRef]

17. Zeng, C.; Richardson, M.; King, D.J. The impacts of environmental variables on water reflectance measured using a lightweight unmanned aerial vehicle (UAV)-based spectrometer system. ISPRS J. Photogramm. Remote Sens. 2017, 130, 217-230. [CrossRef]

18. Field, M.S.; Wilhelm, R.G.; Quinlan, J.F.; Aley, T.J. An assessment of the potential adverse properties of fluorescent tracer dyes used for groundwater tracing. Environ. Monit. Assess. 1995, 38, 75. [CrossRef]

19. Parker, G.G. Tests of Rhodamine WT dye for toxicity to oysters and fish. J. Res. U.S. Geol. Surv. 1973, 1, 499.

20. Rypina, I.I.; Kirincich, A.; Lentz, S.; Sundermeyer, M. Investigating the eddy diffusivity concept in the coastal ocean. J. Phys. Ocean. 2016, 46, 2201-2218. [CrossRef]

21. Feddersen, F.; Olabarrieta, M.; Guza, R.T.; Winters, D.; Raubenheimer, B.; Elgar, S. Observations and modeling of a tidal inlet dye tracer plume. J. Geophys. Res. Oceans. 2016, 121, 7819-7844. [CrossRef]

22. Romero, L.; Ohlmann, J.C.; Palls̀-Sanz, E.; Statom, N.M.; Pérez-Brunius, P.; Maritorena, S. Coincident Observations of Dye and Drifter Relative Dispersion over the Inner Shelf. J. Phys. Oceanogr. 2019, 49, 2447-2468. [CrossRef]

23. Legleiter, C.J.; Manley, P.V.; Erwin, S.O.; Bulliner, E.A. An Experimental Evaluation of the Feasibility of Inferring Concentrations of a Visible Tracer Dye from Remotely Sensed Data in Turbid Rivers. Remote Sens. 2020, 12, 57. [CrossRef]

24. Baek, D.; Seo, I.W.; Kim, J.S.; Nelson, J.M. UAV-based measurements of spatio-temporal concentration distributions of fluorescent tracers in open channel flows. Adv. Water Resour. 2019, 127, 76-88. [CrossRef]

25. Sundermeyer, M.A.; Ledwell, J.R. Lateral dispersion over the continental shelf: Analysis of dye release experiments. J. Geophys. Res. Oceans. 2001, 106, 9603-9621. [CrossRef]

26. Swain, M.J.; Ballard, D.H. Color indexing. Int. J. Comput. Vision 1991, 7, 11-32

27. Shaw, J.A.; Vollmer, M. Blue sun reflected from water: Optical lessons from observations of nature. In Education and Training in Optics and Photonics; Optical Society of America: Washington, DC, USA, 2017; p. 104523B.

28. Lee, Z.; Wang, Y.; Yu, X.; Shang, S.; Luis, K. Evaluation of forward reflectance models and empirical algorithms for chlorophyll concentration of stratified waters. Appl. Opt. 2020, 59, 9340-9352. [CrossRef]

29. Castendyk, D.; Voorhis, J.; Kucera, B. A Validated Method for Pit Lake Water Sampling Using Aerial Drones and Sampling Devices. Mine Water Environ. 2020, 39, 440-454

30. Lee, Z.; Shang, S.; Du, K.; Lin, G.; Liu, T.; Zoffoli, L. Estimating the transmittance of visible solar radiation in the upper ocean using Secchi disk observations. J. Geophys. Res. Oceans 2019, 124, 1434-1444. [CrossRef]

31. Li, Z; Shen, H.; Li, H.; Xia, G.; Gamba, P.; Zhang, L. Multi-feature combined cloud and cloud shadow detection in GaoFen-1 wide field of view imagery. Remote Sens. Environ. 2017, 191, 342-358. [CrossRef]

32. Zhang, L.; Zhang, M.; Sun, X.; Wang, L.; Cen, Y. Cloud removal for hyperspectral remotely sensed images based on hyperspectral information fusion. Int. J. Remote Sens. 2018, 39, 6646-6656. [CrossRef]

33. Lin, H.; Lee, Z.; Lin, G.; Yu, X. Experimental evaluation of the self-shadow and its correction for on-water measurements of water-leaving radiance. Appl. Opt. 2020, 59, 5325-5334. [CrossRef] [PubMed]

34. Socolofsky, S.A.; Adams, E.E.; Sherwood, C.R. Formation dynamics of subsurface hydrocarbon intrusions following the deepwater horizon blowout. Geophys. Res. Lett. 2011, 38. [CrossRef] 